\title{
Prostatectomía radical y calidad de vida: resultados en un centro de alta complejidad
}

\section{Radical Prostatectomy and Quality of Life: Results in a Center of High Complexity}

\author{
Catalina Sánchez Basto ${ }^{1}$ Juan Guillermo Cataño Cataño ${ }^{2}$ Ana carolina Lizcano Herrera ${ }^{3}$ \\ Julián Chavarriaga Soto ${ }^{4}$ Paola Andrea Orrego Rojas ${ }^{3}$ Eliana Samantha Monrroy Feijóo ${ }^{3}$ \\ Nayib Omar Fakih García ${ }^{3}$ Jose Miguel Silva Herrera ${ }^{5}$
}

${ }^{1}$ Uróloga. Pontificia Universidad Javeriana, Bogotá, Colombia
2 Facultad de Medicina Pontificia Universidad Javeriana Urólogo.
Hospital Universitario San Ignacio, Bogotá, Colombia
${ }^{3}$ Médica y cirujana. Pontificia Universidad Javeriana, Bogotá, Colombia
${ }^{4}$ Residente urología. Pontificia Universidad Javeriana, Bogotá, Colombia
${ }^{5}$ Urólogo. Hospital Universitario San Ignacio, Bogotá, Colombia Urol Colomb 2018;27:141-146.

\begin{abstract}
Address for correspondence Julián Chavarriaga, MD, Residente de Urologia de Segundo año, Pontificia Universidad Javeriana, Carrera 4 \#67-20, Bogotá, Colombia (e-mail: chavarriagaj@javeriana.edu.co; chavarriagajulian@gmail.com; chavarriagaj@hotmail.com).
\end{abstract}

\section{Resumen}

\section{Palabras Clave}

- cáncer de próstata

- prostatectomía radical

- calidad de vida

- incontinencia urinaria

- disfunción eréctil

- comorbilidad
Introducción La prostatectomía radical (PR) es el patrón de oro para el manejo quirúrgico con intención curativa del cáncer de próstata. Sin embargo, tiene secuelas importantes como la incontinencia urinaria y la disfunción eréctil. El objetivo de este estudio es describir la prevalencia de las secuelas y el compromiso de calidad de vida de los pacientes llevados a PR abierta en un centro de alta complejidad.

Materiales y Métodos Se revisaron los datos de los pacientes llevados a prostatectomía radical en el Hospital Universitario San Ignacio desde el año 2005 hasta el año 2015. Se seleccionaron aquellos que tuvieran más de 12 meses de postoperatorio, previo consentimiento informado verbal por vía telefónica se aplicó el cuestionario UCLA PCI. La información fue organizada para su análisis en una base de datos construida con ese fin para posterior aplicación de las estadísticas descriptivas que permitieran resumir y describir los resultados.

Resultados Durante los años analizados, se realizaron 231 prostatectomías radicales. Se logró tener contacto con 112 de ellos a los cuales se les aplicó el cuestionario UCLA $\mathrm{PCl}$. En el dominio de función urinaria, $57 \%$ de los pacientes reportan tener "control total de la orina," $53 \%$ nunca tienen escape involuntario de la orina, $82 \%$ no requieren el uso de protectores y $62 \%$ presentan goteo de orina menos de 1 vez por semana. En cuanto a la función sexual, $69,3 \%$ califican su habilidad para tener una erección como pobre o muy pobre, $25 \%$ regular y $7 \%$ buena o muy buena. Para $20 \%$ la erección es suficiente para lograr el coito. Y para $52 \%$ esa función implica un problema en su vida. El impacto en la calidad de vida mostró que un $91,8 \%$ de los pacientes consideran que su salud es en general buena muy buena o excelente y un $70 \%$ no tienen ningún tipo de limitación física. received

September 7, 2017

accepted

November 17, 2017

published online

May 16, 2018
DOI https://doi.org/

$10.1055 / \mathrm{s}-0038-1648213$

ISSN 0120-789X.

eISSN 2027-0119.
Copyright ( 2018 , Sociedad Colombiana License terms de Urología. Publicado por Thieme Revinter Publicações Ltda., Rio de Janeiro, Brazil. Todos los derechos reservados.

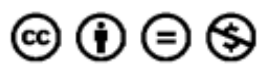




\begin{abstract}
Keywords

- prostate cancer

- radical prostatectomy

- quality of life

- urinary incontinence

- erectile dysfunction

- comorbidity.

Introduction Radical prostatectomy (RP) is the gold standard in curative surgical treatment for prostate cancer. However, it has significant consequences such as urinary incontinence and erectile dysfunction. The aim of this study is to describe the prevalence of complications and its effects on quality of life of patients undergoing an open RP in a reference center.

Materials and Methods The data of patients who underwent RP at Hospital Universitario San Ignacio from 2005 to 2015 was reviewed. Patients who underwent $\mathrm{RP}$ more than 12 months ago where selected and with previous verbal consent a UCLA $\mathrm{PCl}$ questionnaire was completed by phone. The information was organized in a database to apply descriptive statistics which allowed to summarize and analyze the results.

Results During the years analyzed there were performed 231 radical prostatectomies. It was possible to contact 112 by phone to get the UCLA PCI questionnaire completed. In the urinary function domain, $57 \%$ of patients report to "have total control of urine," $53 \%$ never leak, $82 \%$ of them do not require the use of pads and $62 \%$ have urine leakage less than once a week. Regarding sexual function, 69,3\% scored their ability to have an erection as poor or very poor, $25 \%$ medium and $7 \%$ good or very good. For $20 \%$ it was sufficient to achieve coitus. And for $52 \%$ this function means a problem in their life. The impact on life quality showed that $91.8 \%$ of patients felt that their health in general was very good, good or excellent and $70 \%$ did not have any physical limitation.

Conclusions Radical prostatectomy has an important and no insignificant impact in patients' quality of life, especially regarding urinary function and sexual function. It́s impact in quality of life should be considered at the moment of counseling the patients when taking decisions about curative prostate cancer treatment
\end{abstract}

Conclusiones La prostatectomía radical tiene un impacto importante no despreciable en la calidad de vida de los pacientes, especialmente en lo referente a la función urinaria y sexual. Su impacto en la calidad de vida debe ser considerado al momento de realizar la consejería previa a la toma de decisiones en el manejo definitivo del cáncer de próstata con el fin de que el paciente tenga unas expectativas claras en ese sentido.

\section{Introducción}

La prostatectomía radical (PR), es el procedimiento quirúrgico de elección cuando se trata de dar manejo con intención curativa al cáncer de próstata localizado. Fue el primer tratamiento quirúrgico descrito para el cáncer de próstata y ha sido realizado por más de 100 años. Tiene como ventaja importante frente a la radioterapia el permitir la estadificación precisa con el estudio patológico del espécimen quirúrgico; sin embargo, tiene algunas desventajas como la necesidad de hospitalización y recuperación postoperatoria intrahospitalaria, la posibilidad de una resección incompleta y el riesgo de incontinencia urinaria y de disfunción eréctil, entre otras. $^{1-3}$ La frecuencia de complicaciones y secuelas descrita en la literatura es variable, con amplios rangos según las diferentes series y autores disponibles. ${ }^{4-8} \mathrm{Al}$ comparar la PR con otras modalidades de tratamiento curativo, está descrito que la cirugía es la opción terapéutica con mayor riesgo de impotencia e incontinencia, siendo la función sexual la más afectada y la función urinaria e intestinal las menos afectada.9,10

Esas complicaciones, la recuperación postoperatoria y la connotación psicológica que tiene el diagnóstico de cáncer generan cierto grado de afección de la calidad de vida de los pacientes. Por lo anterior, se ha considerado importante evaluar los efectos de los tratamientos y su impacto en la funcionalidad y la calidad de vida. ${ }^{10,11}$

Para esa caracterización, se han creado cuestionarios para la recolección de datos que miden y caracterizan el estado multidimensional de la salud de los pacientes. Esos instrumentos se clasifican en genéricos y específicos. Los primeros, incluyen los puntajes globales, los perfiles de salud y medidas de utilidad, se emplean en diferentes tipos de enfermedades, pacientes o poblaciones y permiten comparar el impacto de las enfermedades, hacer análisis de costoutilidad, obtener valores poblacionales de referencia y evaluar programas para asignar recursos. Los específicos, 
se usan en pacientes o poblaciones para evaluar síntomas, funciones o enfermedades. ${ }^{10,11}$ De esos últimos, existen varios disponibles para cáncer de próstata: Evaluación funcional del tratamiento para cáncer (Functional Assessment of Cancer Therapy: FACT); el índice para cáncer de próstata de la universidad de Californa y Los Angeles (University of California - Los Angeles Prostate Cancer Index: UCLA-PCI) del cual se derivó el Expanded Prostate Index Composite: EPIC y el cuestionario para calidad de vida en investigación y tratamiento de cáncer de próstata europeo (European Organization for Research and Treatment of Cancer Quality of Life Questionnaire: EORTC-QLQ). El UCLA-PCI tiene en cuenta salud en general, función urinaria, función intestinal, función sexual y comorbilidades, es de reconocimiento internacional, validado en idioma español y en nuestro país. ${ }^{12,13}$

El impacto y efectos de los tratamientos tienen el potencial de afectar la calidad de vida, por lo que su éxito debe tener en cuenta la calidad de vida subjetiva y sus secuelas en los pacientes.

Hay múltiples estudios que lo describen alrededor del mundo, sin embargo, pocos en nuestro medio, siendo necesario más estudios para asesorar en la toma de decisiones informadas y para tener estadística local.

El objetivo de este estudio es describir la frecuencia de las complicaciones y el compromiso de calidad de vida de pacientes llevados a PR abierta en el Hospital Universitario San Ignacio durante los últimos 10 años, mediante la aplicación del cuestionario UCLA PCI, con el fin de realizar una descripción de la calidad de vida asociada a la salud y determinar las áreas más afectadas.

\section{Materiales y métodos}

Es un estudio observacional descriptivo de corte transversal. Se realizó una base de datos de los pacientes llevados a PR abierta en el Hospital Universitario San Ignacio desde el año 2005 hasta el 2015.

Se encontró un total de 231 pacientes, de los cuales 112 se lograron contactar telefónicamente, se solicitó consentimiento verbal y se aplicó el cuestionario UCLA - PCI.

El cuestionario UCLA - PCI consiste en 28 preguntas, se divide en 4 secciones que son el estado general del paciente (actividad física diaria básica, laboral o social según el estado físico o emocional, la presencia de dolor y la percepción de salud general actual de cada paciente), la función y molestias urinarias, sexuales e intestinales. Todas las preguntas del cuestionario se basan en hechos ocurridos en las últimas 4 semanas previas a su diligenciamiento. ${ }^{13}$

Los criterios de inclusión fueron: 1 . Pacientes que han sido llevados a prostatectomía radical abierta en el Hospital Universitario San Ignacio desde el año 2005 hasta el año 2015. 2. Llevar más de un año de haber sido llevado a ese procedimiento quirúrgico. 3. Cáncer de próstata localizado o localmente avanzado. Los criterios de exclusión: 1. Pacientes con antecedente de radioterapia. 2. Pacientes con patología neurológica. 3. Cáncer de próstata avanzado. 4. No desear participar en el estudio.
Se analizaron de forma separada los pacientes en tratamiento (farmacológico o quirúrgico) para incontinencia o disfunción eréctil, y en el análisis de la función sexual, se excluyeron aquellos en bloqueo hormonal. El análisis se realizó tabulando los datos en Excel, suma de puntajes para cada dominio y porcentaje de las diferentes variables para su comparación utilizando medidas de frecuencia y tendencia central.

\section{Resultados}

Un total de 231 pacientes fueron llevados a la prostatectomía radical abierta en el HUSI desde el 2005 a 2015. Se logró la aplicación del cuestionario de forma telefónica a 112 pacientes.

Los factores demográficos fueron, edad promedio: 66 años, $0,8 \%$ raza negra y el resto mestizos, $83 \%$ tienen pareja estable, $28 \%$ son universitarios y $40 \%$ bachilleres ( - Tabla 1 ). En cuanto al estadio de la enfermedad, 70\% localizado y $30 \%$ localmente avanzado con la patología inicial, $11 \%$ están en bloqueo hormonal.

En el dominio de función urinaria, $82 \%$ no requieren el uso de protectores, $57 \%$ considera tener un control total de la orina, $62 \%$ presentan goteo de orina menos de 1 vez por semana, $36 \%$ goteo más de una vez por semana y $53 \%$ nunca gotean. Para $16 \%$ la función urinaria es un problema grande en su vida.

Del total, 4,4\% están en tratamiento farmacológico para incontinencia y tan solo un paciente tiene un esfínter artificial. De los que están recibiendo tratamiento farmacológico solo el $20 \%$ afirman tener control total y el paciente del esfínter tiene control total y nunca goteo.

En cuanto a la función sexual (excluyendo a los 11 pacientes en bloqueo hormonal), 69,3\% califican su habilidad para tener una erección como pobre o muy pobre, $25 \%$ regular y $7 \%$ buena o muy buena. Para el $20 \%$ la erección es suficiente para lograr el coito y el $52 \%$ lo considera un problema moderado o grande en su vida.

Tabla 1 Características demográficas

\begin{tabular}{|l|l|}
\hline Característica & Valor $(\boldsymbol{n}=\mathbf{1 1 2})$ \\
\hline Edad promedio (años) & $66(49-85)$ \\
\hline Raza (\%) & $\begin{array}{l}\text { Blanco 23.2 } \\
\text { Negro 0.8 } \\
\text { Latino 7.5 } \\
\text { Multiracial 0.8 }\end{array}$ \\
\hline Relación (\%) & $\begin{array}{l}\text { Vive con esposa o pareja 83 } \\
\text { Tiene una relación, pero no } \\
\text { vive con ella 7.1 } \\
\text { No tiene relación importante 9.8 }\end{array}$ \\
\hline Nivel escolar (\%) & $\begin{array}{l}\text { Menos de bachillerato 31.2 } \\
\text { Bachillerato, técnico 40.1 } \\
\text { Universidad o posgrado 28.5 }\end{array}$ \\
\hline $\begin{array}{l}\text { Trabaja actualmente } \\
\text { (\%) }\end{array}$ & $\begin{array}{l}\text { Sí 51.8 } \\
\text { No 48.1 }\end{array}$ \\
\hline $\begin{array}{l}\text { Estadio patológico } \\
\text { (\%) }\end{array}$ & $\begin{array}{l}\text { Localizado 70 } \\
\text { Localmente avanzado 30 }\end{array}$ \\
\hline Bloqueo hormonal (\%) & 9.82 \\
\hline
\end{tabular}


Del total, $28 \%$ están en tratamiento farmacológico para la disfunción eréctil y de esos 13\% consideran su función sexual buena. Cuatro pacientes fueron sometidos a la colocación de prótesis peneana.

Se evaluó la función sexual ajustada por rangos de edad al momento de la prostatectomía radical sin encontrar diferencia en cuanto al impacto sobre la función sexual.

En cuanto a la función intestinal, un $93 \%$ no tienen ningún tipo de problema con su función intestinal y $0 \%$ incontinencia fecal. El único síntoma reportado es la urgencia fecal en un 14\%.

El impacto en la calidad de vida mostro que un $91,8 \%$ de los pacientes consideran que su salud es en general buena muy buena o excelente y un $70 \%$ no tienen ningún tipo de limitación física.

Los valores por dominios se encuentran en la - tabla número 2.

\section{Discusión}

El objetivo del manejo quirúrgico del cáncer de próstata no está dirigido únicamente a la sobrevida libre de enfermedad, sino también a la preservación de la continencia y la potencia de manera que se afecte de la menor forma posible la calidad de vida del paciente.

La metodología de evaluación de calidad de vida, potencia y continencia es variable y no está estandarizada; por esa razón, las tasas de incontinencia urinaria y de disfunción eréctil son muy variables y hay mucha heterogeneidad en los resultados de los estudios que han evaluado esos desenlaces. ${ }^{4-8,14}$

Las tasas de incontinencia urinaria reportadas en la literatura van desde el $1 \%$ al $20 \%$ y es usual que los hombres presenten incontinencia urinaria transitoria y recuperen la continencia con el paso del tiempo especialmente un año post-tratamiento. ${ }^{4-8}$ La cohorte de 2 años de seguimiento de pacientes llevados a prostatectomía radical de Stanford y col., encontró que solo el 8,4\% de los hombres después de la cirugía tenían incontinencia urinaria y dependiendo de su edad los menores de 75 años tenían tasas hasta de 0,7-3,6\%. ${ }^{4}$ Tasas igualmente bajas encontró Walsh y col. en su estudio de 64 pacientes con 18 meses de seguimiento donde reportó que un $93 \%$ de los pacientes estaban secos y que hasta en un $98 \%$ de ellos, su molestia urinaria era ninguna o pequeña. ${ }^{8}$ Series más recientes y con mayor número de pacientes reportan tasas mayores, la de Haglid y col., con 2431 pacientes de los cuales 778 se llevaron a prostatectomía radical retropúbica, en un seguimiento de 1 año reportó $20,2 \%$ de incontinencia urinaria. ${ }^{6}$ En un estudio local publicado por Cataño y col., entre el 2005 y 2007 en la misma institución del presente estudio, reportaron que un $72 \%$ de los pacientes no requerían ningún tipo de protección urinaria y que un $20 \%$ nunca gotean..$^{13}$

En el presente estudio se encontró que un 18\% requiere el uso de protectores, un $57 \%$ tienen control total de la orina y el $53 \%$ de los pacientes nunca gotean. Esos resultados son similares a las series más grandes y recientes de la literatura mundial. Sin embargo, son mejores al comparar con nuestra estadística local, lo cual podría sugerir que se ha mejorado la técnica quirúrgica y se ha logrado mejor preservación del paquete neurovascular.
En cuanto a disfunción eréctil, las tasas reportadas oscilan entre $14 \%-74 \%{ }^{4-8,14}$ Estudios iniciales como el de Walsh y col., reportó que la recuperación de la función sexual es gradual de tal modo que a los 3 meses el $38 \%$ de los pacientes son potentes, a los 6 meses el $54 \%$, a los 12 meses el $73 \%$ y a los 18 meses el $86 \%$ de los pacientes de su cohorte tienen la potencia conservada, eso traduce que solo el $14 \%$ de los pacientes tienen disfunción eréctil como secuela permanente, sin embargo, todos los pacientes de esa serie eran previamente potentes. ${ }^{8}$ Igualmente, Stanford y col., reporta tasas hasta de 15\%-21\% en menores de 60 años. ${ }^{4}$ Otros estudios más recientes y con mayor número de pacientes encontraron tasas mucho mayores. El estudio de Haglind y col., ya mencionado, encontró tasas de un $74 \%$ de disfunción eréctil, Jackson y col., reporta tasas de impotencia de hasta de un $58,5 \%$ en un seguimiento a 10 años y Ong y col., en 1117 pacientes con seguimiento entre 17-19 meses tasas hasta de $61 \%$ de molestias sexuales de moderadas a grandes..$^{5-7}$ En la serie local de Cataño y col., reportan que un $20 \%$ de los pacientes tiene una erección que logra penetrar y $70 \%$ considera su función sexual pobre. ${ }^{13}$

En nuestro estudio, encontramos que el $70 \%$ de los pacientes refiere que sus erecciones son pobres o muy pobres, $20 \%$ logra penetración suficiente para el coito y el $53 \%$ indican afectación en la calidad de vida. Esos resultados son coherentes con la mayoría de series reportadas en la literatura local e internacional. ${ }^{5-7,13}$ Sin embargo, presenta mayores tasas respecto a lo reportado por Walsh y col. ${ }^{8}$ Esa diferencia se debe principalmente, a factores de selección de los pacientes dado que en dicho estudio todos los pacientes eran potentes antes de la cirugía, y otros factores como la experiencia de los cirujanos y la variabilidad de las formas de medición.

La función intestinal no se ve realmente afectada con la prostatectomía radical, lo cual sigue siendo evidente en la literatura y en el presente estudio. ${ }^{9,13}$

$\mathrm{Al}$ evaluar la calidad de vida asociada a la salud se han descrito varios estudios, con diversos cuestionarios. Se ha reportado que el 78\% de los pacientes escogerían nuevamente el tratamiento con prostatectomía radical y en la mayoría de esos estudios, la satisfacción general varía entre $92 \%-94 \%$, por otra parte, la incontinencia urinaria y disfunción eréctil son los principales predictores de calidad de vida asociada a la salud a los 2 años del procedimiento. ${ }^{15,16}$ En el estudio local encuentran que la percepción general del estado de salud de los pacientes es buena, a pesar de que el 37\% indicaron que empeoró con respecto a la salud previa a la cirugía. ${ }^{13}$ Esas tasas de salud general son compatibles con las encontradas en el presente estudio donde un $91,8 \%$ de los pacientes consideran que su salud es en general buena muy buena o excelente.

Es llamativo que actualmente un $28 \%$ de los pacientes reciben tratamiento farmacológico para disfunción eréctil, un $4,4 \%$ para incontinencia, solo un paciente tiene esfínter urinario y 4 pacientes prótesis peneana. Aunque esos datos no forman parte del objetivo de este estudio, podrían ser útiles para motivar futuros estudios de adherencia, oportunidad y acceso al tratamiento, solicitud por parte del paciente y ofrecimiento por parte del médico tratante. 
Tabla 2 Variables UCLA-PCI

\begin{tabular}{|c|c|c|c|c|}
\hline Dominio & Variables & Frecuencia & Frecuencia relativa & $\mathbf{n}$ \\
\hline \multirow[t]{5}{*}{ Salud general } & Mala & 1 & 0,89 & 112 \\
\hline & Regular & 8 & 7,14 & 112 \\
\hline & Buena & 32 & 28,57 & 112 \\
\hline & Muy buena & 36 & 32,14 & 112 \\
\hline & Excelente & 35 & 31,25 & 112 \\
\hline \multirow[t]{2}{*}{ Limitación por salud física } & Sí & 33 & 29,46 & 112 \\
\hline & No & 79 & 70,53 & 112 \\
\hline \multirow[t]{2}{*}{ Limitación por problemas emocionales } & Sí & 38 & 33,92 & 112 \\
\hline & No & 74 & 66,07 & 112 \\
\hline \multicolumn{5}{|l|}{ Función urinaria } \\
\hline \multirow[t]{4}{*}{ Goteo de orina } & Todos los días & 22 & 19,64 & 112 \\
\hline & 1 vez por semana & 19 & 16,96 & 112 \\
\hline & Menos 1 vez/sem & 11 & 9,82 & 112 \\
\hline & Nunca & 59 & 52,67 & 112 \\
\hline \multirow[t]{3}{*}{ Control de orina } & Total & 64 & 57,14 & 112 \\
\hline & Algún goteo & 44 & 39,28 & 112 \\
\hline & Ningún control & 4 & 3,57 & 112 \\
\hline \multirow[t]{2}{*}{ Uso de protección } & Sí (>0= 1 día) & 20 & 17,85 & 112 \\
\hline & No & 92 & 82,14 & 112 \\
\hline \multirow[t]{2}{*}{ Función urinaria } & Ningún problema o pequeño & 93 & 83,03 & 112 \\
\hline & Moderado o gran problema & 19 & 16,96 & 112 \\
\hline \multicolumn{5}{|l|}{ Pacientes en tratamiento incontinencia } \\
\hline \multicolumn{2}{|l|}{ Farmacológico } & 5 & 4,46 & 112 \\
\hline \multicolumn{2}{|l|}{ Quirúrgico } & 1 & 0,89 & 112 \\
\hline \multicolumn{5}{|l|}{ Función intestinal } \\
\hline \multirow[t]{2}{*}{ Urgencia rectal } & Ha presentado $>1$ vez por semana & 16 & 14,28 & 112 \\
\hline & No ha presentado & 96 & 85,71 & 112 \\
\hline \multirow[t]{2}{*}{ Función intestinal } & Ningún problema o pequeño & 105 & 93,75 & 112 \\
\hline & Moderado o gran problema & 7 & 6,25 & 112 \\
\hline \multicolumn{5}{|c|}{ Función sexual (excluidos 11 pacientes en bloqueo hormonal) } \\
\hline \multirow[t]{3}{*}{ Calificación de la erección } & Pobre o muy pobre & 70 & 69,30 & 101 \\
\hline & Regular & 25 & 24,75 & 101 \\
\hline & Buena o muy buena & 17 & 6,83 & 101 \\
\hline \multirow[t]{2}{*}{ Calidad de la erección } & Suficiente para el coito & 21 & 20,79 & 101 \\
\hline & No suficiente para el coito & 91 & 90,09 & 101 \\
\hline \multirow[t]{2}{*}{ Función sexual } & Ningún problema o pequeño & 59 & 58,41 & 101 \\
\hline & Moderado o gran problema & 53 & 52,47 & 101 \\
\hline \multicolumn{5}{|l|}{ Pacientes en tratamiento para la erección } \\
\hline \multicolumn{2}{|l|}{ Farmacológico } & 29 & 28,71 & 101 \\
\hline \multicolumn{2}{|l|}{ Quirúrgico } & 4 & 3,96 & 101 \\
\hline
\end{tabular}

Los resultados de este estudio son similares a los de las grandes series de pacientes llevados a prostatectomía radical abierta, por otro lado, la mayoría de estudios recientes han demostrado que no hay diferencia en las tasas de incontinencia urinaria ni de disfunción eréctil al comparar la vía abierta con la vía laparoscópica asistida con robot. Un meta análisis del 2012 reportó mejores resultados funcionales con la prostatectomía laparoscópica asistida 
con robot. ${ }^{17,18}$ Sin embargo, un estudio controlado aleatorizado fase 3 que compara cirugía robot asistida con cirugía abierta publicado en 2016 por Yaxley y col., indica que a las 12 semanas las tasas de función sexual y continencia no presentaban diferencia estadísticamente significativa entre las dos técnicas, con lo cual recomiendan a los pacientes optar por un cirujano experimentado en quien confíen antes de decidir una técnica sobre otra. ${ }^{19}$ Apoyando esos resultados está el seguimiento a 10 años de Jackson y col. y el seguimiento a un año de Ong y col. ${ }^{5,7}$

Son debilidades de nuestro estudio, el pequeño tamaño de la muestra y la manera retrospectiva de búsqueda de información y reclutamiento de los pacientes. Sin embargo, se destaca la juiciosa aplicación de escalas validadas y la comparación con una serie similar en el mismo centro.

\section{Conclusiones}

La prostatectomía radical tiene un impacto importante no despreciable en la calidad de vida de los pacientes especialmente en lo referente a función urinaria y función sexual. Eso es evidente en datos locales, así como en la literatura mundial.

El impacto en la calidad de vida de los pacientes llevados a prostatectomía radical debe ser considerado al momento de realizar la consejería previa a la toma de decisiones en el manejo definitivo del cáncer de próstata, con el fin de que el paciente tenga unas expectativas claras en ese sentido.

Conflicto de interés

Declaramos que no existen conflictos de interés en este artículo.

Protección de personas y animales

Los autores declaran que los procedimientos seguidos se conformaron a las normas éticas del comité de experimentación humana responsable y de acuerdo con la Asociación Médica Mundial y la Declaración de Helsinki.

Confidencialidad de los datos

Los autores declaran que han seguido los protocolos de su centro de trabajo sobre la publicación de datos de pacientes.

Derecho a la privacidad y consentimiento informado Los autores han obtenido el consentimiento informado de los pacientes y/o sujetos referidos en el artículo. Este documento obra en poder del autor de correspondencia.

\section{Referencias}

1 Hull GW, Rabbani F, Abbas F, Wheeler TM, Kattan MW, Scardino PT. Cancer control with radical prostatectomy alone in 1,000 consecutive patients. J Urol 2002;167(2 Pt 1):528-534
2 Pound CR, Partin AW, Eisenberger MA, Chan DW, Pearson JD, Walsh PC. Natural history of progression after PSA elevation following radical prostatectomy. JAMA 1999;281(17):1591-1597

3 Kupelian P, Katcher J, Levin H, Zippe C, Klein E. Correlation of clinical and pathologic factors with rising prostate-specific antigen profiles after radical prostatectomy alone for clinically localized prostate cancer. Urology 1996;48(02):249-260

4 Stanford JL, Feng Z, Hamilton AS, y col. Urinary and sexual function after radical prostatectomy for clinically localized prostate cancer: the Prostate Cancer Outcomes Study. JAMA 2000;283(03):354-360

5 Jackson MA, Bellas N, Siegrist T, y col. Experienced Open vs Early Robotic-assisted Laparoscopic Radical Prostatectomy: A 10-year Prospective and Retrospective Comparison. Urology 2016;91:111-118

6 Haglind E, Carlsson S, Stranne J, et al; LAPPRO steering committee. Urinary Incontinence and Erectile Dysfunction After Robotic Versus Open Radical Prostatectomy: A Prospective, Controlled, Nonrandomised Trial. Eur Urol 2015;68(02):216-225

7 Ong WL, Evans SM, Spelman T, Kearns PA, Murphy DG, Millar JL. Comparison of oncological and health-related quality of life outcomes between open and robot-assisted radical prostatectomy for localised prostate cancer - findings from the population-based Victorian Prostate Cancer Registry. BJU Int 2016;118(04):563-569

8 Walsh PC, Marschke P, Ricker D, Burnett AL. Patient-reported urinary continence and sexual function after anatomic radical prostatectomy. Urology 2000;55(01):58-61

9 Pardo Y, Guedea F, Aguiló F, y col. Quality-of-life impact of primary treatments for localized prostate cancer in patients without hormonal treatment. J Clin Oncol 2010;28(31):4687-4696

10 Namiki S, Satoh T, Baba S, y col. Quality of life after brachytherapy or radical prostatectomy for localized prostate cancer: a prospective longitudinal study. Urology 2006;68(06):1230-1236

11 Zúniga MA, Carrillo-Jiménez GT, Fos PJ, Gandek B, Medina-Moreno MR. Evaluación del estado de salud con la Encuesta SF-36: resultados preliminares en México. Salud Publica Mex 1999;41(02):110-118

12 Krongrad A, Perczek RE, Burke MA, Granville LJ, Lai H, Lai S. Reliability of Spanish translations of select urological quality of life instruments. J Urol 1997;158(02):493-496

13 Cataño J, Morales C. Evaluación De La Calidad De Vida Asociada a La Salud En Los Pacientes Sometidos a Prostatectomia Radical Abierta Por Carcinoma De Próstata Clinicamente Localizado. Rev Colomb Urol. 2009;XVIII(03):22-30

14 Catalona WJ, Basler JW. Return of erections and urinary continence following nerve sparing radical retropubic prostatectomy. J Urol 1993;150(03):905-907

15 Prabhu V, Lee T, McClintock TR, Lepor H. Short-, Intermediate-, and Long-term Quality of Life Outcomes Following Radical Prostatectomy for Clinically Localized Prostate Cancer. Rev Urol 2013;15(04):161-177

16 Huang GJ, Sadetsky N, Penson DF. Health related quality of life for men treated for localized prostate cancer with long-term followup. J Urol 2010;183(06):2206-2212

17 Novara G, Ficarra V, Mocellin S, y col. Systematic review and metaanalysis of studies reporting oncologic outcome after robotassisted radical prostatectomy. Eur Urol 2012;62(03):382-404

18 Ficarra V, Novara G, Ahlering TE, y col. Systematic review and meta-analysis of studies reporting potency rates after robotassisted radical prostatectomy. Eur Urol 2012;62(03):418-430

19 Yaxley JW, Coughlin GD, Chambers SK, y col. Robot-assisted laparoscopic prostatectomy versus open radical retropubic prostatectomy: early outcomes from a randomised controlled phase 3 study. Lancet 2016;388(10049):1057-1066 